\title{
A New Definition of Fractional Derivatives Based on Truncated Left-Handed Grünwald-Letnikov Formula with $0<\alpha<1$ and Median Correction
}

\author{
Zhiwu Liao \\ School of Computer Science, Sichuan Normal University, Chengdu, Sichuan 610101, China \\ Correspondence should be addressed to Zhiwu Liao; liaozhiwu@163.com
}

Received 10 February 2014; Accepted 23 March 2014; Published 13 April 2014

Academic Editor: Ming Li

Copyright (c) 2014 Zhiwu Liao. This is an open access article distributed under the Creative Commons Attribution License, which permits unrestricted use, distribution, and reproduction in any medium, provided the original work is properly cited.

\begin{abstract}
We propose a new definition of fractional derivatives based on truncated left-handed Grünwald-Letnikov formula with $0<\alpha<1$ and median correction. Analyzing the difficulties to choose the fractional orders and unsatisfied processing results in signal processing using fractional-order partial differential equations and related methods; we think that the nonzero values of the truncated fractional order derivatives in the smooth regions are major causes for these situations. In order to resolve the problem, the absolute values of truncated parts of the G-L formula are estimated by the median of signal values of the remainder parts, and then the truncated G-L formula is modified by replacing each of the original signal value to the differences of the signal value and the median. Since the sum of the coefficients of the G-L formula is zero, the median correction can reduce the truncated errors greatly to proximate G-L formula better. We also present some simulation results and experiments to support our theory analysis.
\end{abstract}

\section{Introduction}

Partial differential equations (PDEs) and related methods are very important tools for signal processing [1-13]. Especially, in recent years progress was achieved in the theory of fractional calculus as a useful tool to handle applications in the area of physics, chemistry, and engineering sciences [620].

However, unlike integer-order derivatives with zeros or small values in the smooth regions and with big values near singularities, the values of truncated fractional derivatives are with very large absolute values. The direct result is that the derivative values cannot be used to measure the degrees of singularities. That is, small derivative values perhaps relate to singularity regions and big derivative values perhaps relate to smooth regions. Therefore, some integer-order PDE methods cannot be modified to their fractional-order counterparts directly. Hu noticed the problem in 2013 and proposed a new fractional-order PDE with different orders of different parts for fractional-order PM method [13]. However, he did not give the analysis in theory.
In this paper, we study behaviors of fractional-order derivatives of truncated left-handed Grünwald-Letnikov formula with $0<\alpha<1$. Based on the analysis, a new fractional derivative formula is proposed based on truncated left-handed Grünwald-Letnikov formula with $0<\alpha<1$ and median correction. The median correction is used to reduce the truncated errors of G-L formula.

The rest of this paper is as follows. Section 2 introduces some basic theory backgrounds in fractional derivatives; we also deduce some useful results based on these theory backgrounds. In Section 3 we introduce the truncated G-L formula and its numerical approximation. Section 4 presents the new fractional derivative formula and gives properties and numerical methods for the new fractional definition. The simulation results and experiments are presented in Section 5. We also give conclusions and acknowledgments finally.

\section{Fractional Derivatives}

In this section, we will introduce some preparations for the new method, that is, Grünwald-Letnikov formula and its matrix approximation. 
2.1. Grünwald-Letnikov Formula. Fractional-order derivatives are defined as operators whose orders have been extended to noninteger numbers. There are a number of definitions of fractional derivatives. One usual way of representing the discrete fractional derivatives is by the GrünwaldLetnikov (G-L) formula $[21,22]$, which is

$$
D_{\mathrm{GL}}^{\alpha} u(x)=\lim _{\Delta x \rightarrow 0} \frac{1}{\Delta x^{\alpha}} \sum_{k=0}^{[(x-a) / \Delta x]} \omega_{k}^{(\alpha)} u(x-k \Delta x)
$$

where $x \in[a, b], \Delta x$ denotes the uniform space step, and $\omega_{k}^{(\alpha)}=(-1)^{k}\left(\begin{array}{l}\alpha \\ k\end{array}\right)$ represents the normalized G-L weights which are given by

$$
\begin{gathered}
\omega_{0}^{(\alpha)}=1, \\
\omega_{k}^{(\alpha)}=(-1)^{k} \frac{\alpha(\alpha-1) \cdots(\alpha-k+1)}{k !}=\frac{\Gamma(k-\alpha)}{\Gamma(-\alpha) \Gamma(k+1)}, \\
\text { for } k=1,2,3, \ldots
\end{gathered}
$$

For $\alpha=1$, (1) becomes the classical 1st derivative, and for any $\alpha=n, n \in \aleph$ is a positive integer; they are classical $n$th derivatives of $u(x)$. Note that for when $\alpha$ is a positive integer, equations are with limit support whose support lengths are $\alpha+1$. However, for when $\alpha$ is not an integer, fractional derivatives are nonlocal operators. That is, the value of the fractional derivative at a point $x$ depends on the function values at all the points to the left of the point $x$.

Therefore, in order to handle fractional derivative numerically, it is necessary to compute the coefficients $\omega_{k}^{(\alpha)}$, where $\alpha$ is the order of the fractional derivative. For that we can use the recurrence relationships:

$$
\begin{gathered}
\omega_{0}(\alpha)=1 ; \\
\omega_{k}^{(\alpha)}=\left(1-\frac{\alpha+1}{k}\right) \omega_{k-1}^{(\alpha)}, \quad k=1,2,3, \ldots
\end{gathered}
$$

Some useful properties for left-handed G-L formula are presented as follows.

Lemma 1. The nonlocal operator defined in (1) is a linear operator.
Proof. $u(x)$ and $v(x)$ are two functions, and $x \in[a, b] ; \lambda$ is a real number. We have

(1)

$$
\begin{aligned}
& D_{\mathrm{GL}}^{\alpha}[u(x)+v(x)] \\
& =\lim _{\Delta x \rightarrow 0} \frac{1}{\Delta x^{\alpha}} \\
& \quad \times \sum_{k=0}^{[(x-a) / \Delta x]} \omega_{k}^{(\alpha)}[u(x-k \Delta x)+v(x-k \Delta x)] \\
& =\lim _{\Delta x \rightarrow 0} \frac{1}{\Delta x^{\alpha}} \sum_{k=0}^{[(x-a) / \Delta x]} \omega_{k}^{(\alpha)} u(x-k \Delta x) \\
& +\lim _{\Delta x \rightarrow 0} \frac{1}{\Delta x^{\alpha}} \sum_{k=0}^{[(x-a) / \Delta x]} \omega_{k}^{(\alpha)} v(x-k \Delta x) \\
& =D_{\mathrm{GL}}^{\alpha} u(x)+D_{\mathrm{GL}}^{\alpha} v(x)
\end{aligned}
$$

(2) $D_{\mathrm{GL}}^{\alpha}[\lambda u(x)]=\lim _{\Delta x \rightarrow 0} \frac{1}{\Delta x^{\alpha}} \sum_{k=0}^{[(x-a) / \Delta x]} \omega_{k}^{(\alpha)}[\lambda u(x)]$

$$
\begin{aligned}
& =\lambda \lim _{\Delta x \rightarrow 0} \frac{1}{\Delta x^{\alpha}} \sum_{k=0}^{[(x-a) / \Delta x]} \omega_{k}^{(\alpha)} u(x) \\
& =\lambda D_{\mathrm{GL}}^{\alpha} u(x) .
\end{aligned}
$$

Lemma 2. $\sum_{k=0}^{\infty} \omega_{k}^{(\alpha)}=0$

Proof. Since $(1-z)^{\alpha}=\sum_{k=0}^{\infty} \omega_{k}^{(\alpha)} z^{k}$, let $k=1$; we have

$$
\sum_{k=0}^{\infty} \omega_{k}^{(\alpha)}=(1-1)^{\alpha}=0
$$

Lemma 3. For $0<\alpha<1, m$ is a positive integer; one has

$$
\begin{gathered}
\omega_{k}^{(\alpha)}<0, \quad k=1,2,3, \ldots, \\
\sum_{k=m}^{\infty} \omega_{k}^{(\alpha)}<0 .
\end{gathered}
$$

Proof. For $k=1, \omega_{1}^{(\alpha)}=-\alpha<0$.

Assume that $\omega_{k}^{(\alpha)}<0$.

According to (3), we have $\omega_{k+1}^{(\alpha)}=(1-(\alpha+1) /(k+1)) \omega_{k}^{(\alpha)}$. Since $0<\alpha<1$ and $k+1 \geq 2,(1-(\alpha+1) /(k+1))>0$. Thus, $\operatorname{sgn}\left(\omega_{k+1}^{(\alpha)}\right)=\operatorname{sgn}\left(\omega_{k}^{(\alpha)}\right)<0$. Here

$$
\operatorname{sgn}(x)= \begin{cases}1, & x \geq 0 \\ -1, & x<0\end{cases}
$$

Then, we have $\omega_{k+1}^{(\alpha)}<0$. Thus, $\sum_{k=m}^{\infty} \omega_{k}^{(\alpha)}<0$. 
Lemma 4. For $0<\alpha<1, m \geq 0$ is an integer. One has

$$
\sum_{k=m}^{\infty} \omega_{k}^{(\alpha)}(-k)>0 \text {. }
$$

Proof. From Lemma 3, for $0<\alpha<1, \omega_{k}^{(\alpha)}<0, k=1,2,3, \ldots$. Thus, $\omega_{k}^{(\alpha)}(-k)>0$ for $k=1,2,3, \ldots$. We have $\sum_{k=m}^{\infty} \omega_{k}^{(\alpha)}(-k)>0, m \geq 1$.

Since $\omega_{0}^{(\alpha)}(-0)=0$, we have $\sum_{k=m}^{\infty} \omega_{k}^{(\alpha)}(-k)>0, m \geq 0$.

2.2. Numerical Method of G-L Formula. For G-L formula in (1) in signal processing, the uniform space step is set to $\Delta x=1$ for easy description; $x$ is the variant whose support is $[a, b]$. That is, the signal $u(x)$ is compact support. Therefore, equations can be specified as

$$
D_{\mathrm{GL}}^{\alpha}=\sum_{k=0}^{[x-a]} \omega_{k}^{(\alpha)} s(x-k) .
$$

The coefficients can also be obtained recursively from (3). We can discretize (1) into a finite difference on a grid on the $x$ axis, where the 0 th lattice is $a$; $j$ th lattice is $x_{j}, j=1,2, \ldots, n-$ 1 ; and the $n$th lattice is $b$. That is, $n$ is the length of the signal.

Therefore, using the matrix approximate method, we have

$$
D_{\mathrm{GL}}^{\alpha} \mathbf{S}=M^{(\alpha)} \mathbf{S},
$$

where TGL represents truncated G-L formula, $\mathbf{S}=\left[s\left(x_{0}\right)\right.$, $\left.s\left(x_{1}\right), \ldots, s\left(x_{n}\right)\right]^{T}, T$ represents the transposed vector, and $M^{(\alpha)}$ is an $n \times n$ lower triangular strip matrix defined as

$$
M^{(\alpha)}=\left(\begin{array}{cccc}
\omega_{0}^{(\alpha)} & 0 & \ldots & 0 \\
\omega_{1}^{(\alpha)} & \omega_{0}^{(\alpha)} & \ldots & \vdots \\
\vdots & \vdots & \ddots & 0 \\
\omega_{n}^{(\alpha)} & \omega_{n-1}^{(\alpha)} & \cdots & \omega_{0}^{(\alpha)}
\end{array}\right) .
$$

\section{Truncated Grünwald-Letnikov Formula}

Fractional integration and fractional differentiation are generalizations of notions of integer-order integration and differentiation and include $n$th derivatives ( $n$ denotes an integer number) as particular cases. One usual way of representing the discrete fractional derivatives is by the GrünwaldLetnikov (G-L) formula introduced in Section 2 (see (1)).

However, for digital signals, we have to discuss truncated G-L formula rather than the G-L formula itself because of the limited supports of digital signals. In this section, the definition of truncated G-L formula and its properties are discussed firstly and then we will give its numerical scheme.

3.1. Truncated Grünwald-Letnikov Formula. The truncated $\mathrm{G}-\mathrm{L}$ formula is

$$
D_{\mathrm{TGL}}^{\alpha} s(x)=\sum_{k=0}^{t} \omega_{k}^{(\alpha)} s(x-k),
$$

where $x \in[a, b]$, the uniform space step is set to $1, t$ is the length of the support, $\omega_{k}^{(\alpha)}=(-1)^{k}\left(\begin{array}{c}\alpha \\ k\end{array}\right)$ represents the normalized G-L weights, and their recurrence relationship is given by (3).

Just as above sections, we will discuss some properties of truncated G-L formula.

Lemma $2^{\prime}$. For $0<\alpha<1, t \geq 0$ is an integer. One has

$$
\sum_{k=0}^{t} \omega_{k}^{(\alpha)}>0
$$

Proof. According to Lemma 2, we have

$$
0=\sum_{k=0}^{\infty} \omega_{k}^{(\alpha)}=\sum_{k=0}^{t} \omega_{k}^{(\alpha)}+\sum_{k=t+1}^{\infty} \omega_{k}^{(\alpha)} .
$$

According to Lemma $3, \sum_{k=t+1}^{\infty} \omega_{k}^{(\alpha)}<0$. Therefore, $\sum_{k=0}^{t} \omega_{k}^{(\alpha)}>0$.

Lemma $3^{\prime}$. For $0<\alpha<1, m$ is a positive integer, $t \geq m$. One has

$$
\sum_{k=m}^{t} \omega_{k}^{(\alpha)}<0
$$

Proof. For $k=1, \omega_{1}^{(\alpha)}=-\alpha<0$.

Assume that $\omega_{k}^{(\alpha)}<0$.

According to Lemma 1 , we have $\omega_{k+1}^{(\alpha)}=(1-(\alpha+1) /(k+$ 1)) $\omega_{k}^{(\alpha)}$. Since $0<\alpha<1$ and $k+1 \geq 2,(1-(\alpha+1) /(k+1))>0$. Thus, $\operatorname{sgn}\left(\omega_{k+1}^{(\alpha)}\right)=\operatorname{sgn}\left(\omega_{k}^{(\alpha)}\right)<0$. Here

$$
\operatorname{sgn}(x)= \begin{cases}1, & x \geq 0, \\ -1, & x<0 .\end{cases}
$$

Then, we have $\omega_{k+1}^{(\alpha)}<0$. Thus, $\sum_{k=m}^{t} \omega_{k}^{(\alpha)}<0$.

Lemma $4^{\prime}$. For $0<\alpha<1, m \geq 0$ is an integer and $t$ is a positive integer. One has

$$
\sum_{k=m}^{t} \omega_{k}^{(\alpha)}(-k)>0
$$

Proof. From Lemma 3, for $0<\alpha<1, \omega_{k}^{(\alpha)}<0$, $k=$ $1,2,3, \ldots$.

Thus, $\omega_{k}^{(\alpha)}(-k)>0$ for $k=1,2,3, \ldots, t$. We have $\sum_{k=m}^{t} \omega_{k}^{(\alpha)}(-k)>0, \quad m \geq 0, \quad t \geq 1$.

3.2. Numerical Method of Truncated G-L Formula. We can discretize (12) into a finite difference on a grid on the $x$ axis, where the 0th lattice is $a$; $j$ th lattice is $x_{j}, j=1,2, \ldots, n-1$; and the $n$th lattice is $b$. That is, $n$ is length of signals.

Therefore, using the matrix approximate method, we have

$$
D_{\mathrm{GL}}^{\alpha} \mathbf{S} \approx D_{\mathrm{TGL}}^{\alpha} \mathbf{S}=M_{T}^{(\alpha)} \mathbf{S}
$$


where $\mathbf{S}=\left[s\left(x_{0}\right), s\left(x_{1}\right), \ldots, s\left(x_{n}\right)\right]^{T}, T$ represents the transposed vector, and $M_{T}^{(\alpha)}$ is an $n \times n$ matrix defined as

$$
M_{T}^{(\alpha)}=\left(\begin{array}{ccccccc}
\omega_{0}^{(\alpha)} & 0 & \cdots & 0 & 0 & \cdots & 0 \\
\omega_{1}^{(\alpha)} & \omega_{0}^{(\alpha)} & \ldots & \vdots & 0 & \cdots & 0 \\
\vdots & \vdots & \ddots & 0 & \vdots & \vdots & \\
\omega_{t}^{(\alpha)} & \omega_{t-1}^{(\alpha)} & \cdots & \omega_{0}^{(\alpha)} & 0 & \cdots & 0 \\
0 & \omega_{t}^{(\alpha)} & \omega_{t-1}^{(\alpha)} & \cdots & \omega_{0}^{(\alpha)} & \cdots & 0 \\
\vdots & \vdots & \ddots & \ddots & \ddots & \vdots & \\
0 & \cdots & 0 & \omega_{t}^{(\alpha)} & \omega_{t-1}^{(\alpha)} & \cdots & \omega_{0}^{(\alpha)}
\end{array}\right)
$$

Notice that $n$ is the length of the signal and $t$ is the length of the support. That is, $n \geq t$. Since $M_{T}^{(\alpha)}$ is sparser than $M^{(\alpha)}$ for $n>t$, the computation cost of the truncated G-L formula is lower than that of the G-L formula.

\section{New Truncated Grünwald-Letnikov Formula}

Although Lemma 2 tells us the values of fractional derivatives for a constant function $s(x)=1$ defined by G-L formula equal to zeros, for truncated G-L formula, Lemma $2^{\prime}$ shows that it is not true.

The main awkwardness for this situation comparing with integer-order derivatives is that the fractional derivatives cannot be used to measure the strength of singularities. Therefore, estimation methods based on the strength of singularities measured by the modula of 1-order derivatives cannot be generalized to their fractional counterparts directly. These estimation methods include many popular and state-of-art frameworks, such as anisotropic diffusion, nonlocal means, and bilateral filtering.

Therefore, in order to generalize fractional derivatives to these frameworks, the truncated G-L formula should be modified as follows: for $s(x)=c, c \neq 0, D^{\alpha} s(x)=0$.

We start from the requirement to obtain the definition and properties of the new truncated G-L formula, and then the numerical method of the new model by matrix is presented.

4.1. Motivations and Definitions. The discussion is from the error of truncated G-L formula.

Definition 5. The error of the truncated G-L formula is

$$
\begin{aligned}
\operatorname{Err}_{\mathrm{TGL}}(s(x)) & =\sum_{k=0}^{\infty} \omega_{k}^{(\alpha)} s(x-k)-\sum_{k=0}^{t} \omega_{k}^{(\alpha)} s(x-k) \\
& =\sum_{k=t+1}^{\infty} \omega_{k}^{(\alpha)} s(x-k)
\end{aligned}
$$

where TGL represents truncated G-L formula, $t$ is the support length, and $s(\cdot)$ is the signal, $0<\alpha<1$.
Especially, for $s(x)=1$, we have

$$
\operatorname{Err}_{\mathrm{TGL}}(s(x))=\sum_{k=t+1}^{\infty} \omega_{k}^{(\alpha)}
$$

that is, the sum of all terms after $t$. From Lemma $2^{\prime}$, we have $\sum_{k=0}^{t} \omega_{k}^{(\alpha)}>0$, for $t \geq 0,0<\alpha<1$ and from Lemma 3, we have $\omega_{k}^{(\alpha)}<0$, for $k=1,2, \ldots, 0<\alpha<1$, which implies that the error will become smaller as $t$ becomes bigger.

Moreover, from Lemma 2, we have $\sum_{k=0}^{\infty} \omega_{k}^{(\alpha)}=0$; thus, the truncated error can be changed as follows.

Proposition 6. The truncated error of $s(x)=1$ is

$$
\operatorname{Err}_{T G L}(s(x))=-\sum_{k=0}^{t} \omega_{k}^{(\alpha)} \text {. }
$$

Proof. From Lemma 2 and the above equation,

$$
\begin{aligned}
0 & =\sum_{k=0}^{\infty} \omega_{k}^{(\alpha)}=\sum_{k=0}^{t} \omega_{k}^{(\alpha)}+\sum_{k=t+1}^{\infty} \omega_{k}^{(\alpha)} \\
& =\sum_{k=0}^{t} \omega_{k}^{(\alpha)}+\operatorname{Err}_{\mathrm{TGL}}(s(x)) .
\end{aligned}
$$

Thus,

$$
\operatorname{Err}_{\mathrm{TGL}}(s(x))=-\sum_{k=0}^{t} \omega_{k}^{(\alpha)}
$$

For $s(x)=c, c$ is a constant real number; we can get similar results.

Proposition 7. The truncated error of $s(x)=c \neq 0$ is

$$
\operatorname{Err}_{T G L}(s(x))=-c \sum_{k=0}^{t} \omega_{k}^{(\alpha)} .
$$

Similarly, for $s(x) \neq$ constant, values from $t+1$ to $\infty$ are not known and we only know the samples from 0 to $t$. Thus, we should estimate errors defined in (20) by the values from 0 to $t$. For $s(x)=$ constant, estimate errors defined in (20) by the values from 0 to $t$ have been accomplished through Proposition 7, which reminds us that the problem can be solved by assuming $s(x)$ a constant. Thus, times the constant and the sum of $\omega_{k}^{(\alpha)}, k=0,1, \ldots, k$ can approximate the error well. This constant also should be estimated from values from 0 to $t$.

One alternative scheme is that the median of values from 0 to $t$ is used as the estimate value for the constant since median is an estimate with good performance in flexibility and reliability.

Definition 8. The new truncated G-L formula is

$$
D_{\mathrm{NTGL}}^{\alpha} s(x)=\sum_{k=0}^{t} \omega_{k}^{(\alpha)} s(x-k)-\operatorname{median}(\mathbf{S}) \sum_{k=0}^{t} \omega_{k}^{(\alpha)},
$$


where NTGL represents the new truncated G-L formula, $\operatorname{median}(\mathbf{S})$ is the median of the vector $\mathbf{S}=[s(x), s(x-$ $1), \ldots, s(x-t)]^{T}$, the uniform space step is set to $1, t$ is the length of the support, and $\omega_{k}^{(\alpha)}=(-1)^{k}\left(\begin{array}{l}\alpha \\ k\end{array}\right)$ represents the normalized G-L weights.

4.2. Numerical Method of New Truncated G-L Formula. We can discretize (26) into a finite difference on a grid on the $x$ axis, where the 0th lattice is $a$; $j$ th lattice is $x_{j}, j=1,2, \ldots, n-$ 1 ; and the $n$th lattice is $b$. That is, $n$ is length of signals.

Therefore, using the matrix approximate method, we have

$$
D_{\mathrm{GL}}^{\alpha} \mathbf{S} \approx D_{\mathrm{NTGL}}^{\alpha} \mathbf{S}_{\mathbf{c}}=M_{T} \mathbf{S}_{\mathrm{c}}
$$

where NTGL represents new truncated G-L formula, $\mathbf{S}=$ $\left[s\left(x_{0}\right), s\left(x_{1}\right), \ldots, s\left(x_{n}\right)\right]^{T}$, and $T$ represents the transposed vector. $\mathbf{S}_{\mathbf{c}}=\left[s\left(x_{0}\right)-\operatorname{median}(\mathbf{S}), s\left(x_{1}\right)-\operatorname{median}(\mathbf{S}), \ldots, s\left(x_{n}\right)-\right.$ $\operatorname{median}(\mathbf{S})]^{T}$ is the corrected vector of $\mathbf{S}$ by the median of $\mathbf{S}$, and $M_{T}$ is an $n \times n$ matrix defined as

$$
M_{T}=\left(\begin{array}{ccccccc}
\omega_{0}^{(\alpha)} & 0 & \ldots & 0 & 0 & \cdots & 0 \\
\omega_{1}^{(\alpha)} & \omega_{0}^{(\alpha)} & \ldots & \vdots & 0 & \cdots & 0 \\
\vdots & \vdots & \ddots & 0 & \vdots & \vdots & \\
\omega_{t}^{(\alpha)} & \omega_{t-1}^{(\alpha)} & \cdots & \omega_{0}^{(\alpha)} & 0 & \cdots & 0 \\
0 & \omega_{t}^{(\alpha)} & \omega_{t-1}^{(\alpha)} & \cdots & \omega_{0}^{(\alpha)} & \cdots & 0 \\
\vdots & \vdots & \ddots & \ddots & \ddots & \vdots & \\
0 & \cdots & 0 & \omega_{t}^{(\alpha)} & \omega_{t-1}^{(\alpha)} & \cdots & \omega_{0}^{(\alpha)}
\end{array}\right)
$$

Notice that $n$ is the length of the signal and $t$ is the length of the support. That is, $n \geq t$. Since $M_{T}$ is sparser than $M$ for $n>t$, the computation cost of the truncated G-L formula is lower than the G-L formula's.

4.3. Properties of the New Truncated G-L Formula. In this subsection, we will give some important properties of the new truncated G-L formula.

Theorem 9. The nonlocal operator defined in (27) is a linear operator.
Proof. $u(x)$ and $v(x)$ are two functions, and $x \in[a, b] ; \lambda$ is a real number. We have

(1)

$$
\begin{aligned}
& D_{\mathrm{NTGL}}^{\alpha}[u(x)+v(x)]=D_{\mathrm{NTGL}}^{\alpha}[\mathbf{U}+\mathbf{V}] \\
& =M_{T}\left[\mathbf{U}_{\mathbf{c}}+\mathbf{V}_{\mathbf{c}}\right] \\
& =M_{T} \mathbf{U}_{\mathbf{c}}+M_{T} \mathbf{V}_{\mathbf{c}} \\
& =D_{\mathrm{NTGL}}^{\alpha} u(x)+D_{\mathrm{NTGL}}^{\alpha} v(x), \\
& \begin{aligned}
D_{\mathrm{NTGL}}^{\alpha}[\lambda u(x)] & =D_{\mathrm{NTGL}}^{\alpha}[\lambda \mathbf{U}] \\
& =\lambda M_{T} \mathbf{U}_{\mathbf{c}} \\
& =\lambda D_{\mathrm{NTGL}}^{\alpha} u(x) .
\end{aligned}
\end{aligned}
$$

Here, $\mathbf{U}_{\mathfrak{c}}, \mathbf{V}_{\mathbf{c}}$ are corrected vectors of $\mathbf{U}, \mathbf{V}$, which are defined as the corrected vector in (27).

Theorem 10. $M_{T}^{(\alpha)}$ and $M_{T}^{(\beta)}$ are two matrixes defined in (27), that is, the approximation matrixes of truncated $G-L$ formula with fractional orders $\alpha$ and $\beta$, respectively. Thus,

$$
M_{T}^{(\alpha)} M_{T}^{(\beta)}\left(\begin{array}{ccccccc}
\gamma_{0} & 0 & \ldots & 0 & 0 & \cdots & 0 \\
\gamma_{1} & \gamma_{0} & \ldots & \vdots & 0 & \cdots & 0 \\
\vdots & \vdots & \ddots & 0 & \vdots & \vdots & \\
\gamma_{t} & \gamma_{t-1} & \cdots & \gamma_{0} & 0 & \cdots & 0 \\
0 & \gamma_{t} & \gamma_{t-1} & \cdots & \gamma_{0} & \cdots & 0 \\
\vdots & \vdots & \ddots & \ddots & \ddots & \vdots & \\
0 & \cdots & 0 & \gamma_{t} & \gamma_{t-1} & \cdots & \gamma_{0}
\end{array}\right),
$$

where $\gamma_{i}=\sum_{k=0}^{i} \omega_{k}^{(\alpha)} \omega_{i-k}^{(\beta)}, i=0,1, \ldots, t$.

Lemma 12 can be proved easily by times two matrixes. That is, two operators of the truncated G-L formula with different fractional orders are commutative.

We guess the following equations in Guess 1 are correct. However, we cannot prove it.

Guess 1. We have

$$
D_{\mathrm{NTGL}}^{\alpha} D_{\mathrm{NTGL}}^{\beta} s(x)=D_{\mathrm{NTGL}}^{\beta} D_{\mathrm{NTGL}}^{\alpha} s(x)=D_{\mathrm{NTGL}}^{\alpha+\beta} s(x) .
$$

\section{Numerical Simulations}

For the numerical approximation, although longer memory is more precise computation for the fractional derivatives, a fixed number for $t$ is adopted for reducing computation complexity, for example, $t=100$ or $t=1000$ and so forth. But these truncated forms will lead to some unsatisfied results. In this section, we will give error analysis of truncated G$\mathrm{L}$ formulas firstly and then present experiments using test signals. 
5.1. Error Analysis of Truncated G-L Formula. The error analysis of truncated G-L formula is very important in the applications of fractional derivatives. Some efforts discuss the problem in theory [23]. In this subsection we will discuss the truncated errors by considering the signal $s(x)=1$ since the most serious effect of truncated errors is that the values of fractional derivatives are not equal to zeros when $s(x)=$ constant.

According to Lemma 2, the values of untruncated G-L formula for $s(x)=1$ are equal to zeros, which are coincident to the 1-order derivatives. For the truncated G-L formula when fractional order $\alpha$ satisfies $0<\alpha<1$, according to Lemma $2^{\prime}$, the remainder part of $s(x)=1$ is more than zero. Thus, the truncated part is less than zero. That is, it is a negative number. The truncated errors of truncated GL formula for $s(x)=1$ with different support lengths and different fractional-orders $\alpha, 0<\alpha<1$, are shown in Table 1 , which demonstrate the above conclusions.

Moreover, we will compare the changes of absolute values of truncated errors with different $\alpha$ and different support lengths.

Lemma 11. The absolute values of truncated errors | $\sum_{k=0}^{t} \omega_{k}^{(\alpha)} \mid$, where $t$ is the support length, for $s(x)=1$, become smaller as the support lengths become larger.

Proof. According to Lemma 3, $\omega_{k}^{(\alpha)}<0,0<\alpha<1$, for $k \leq 1$ is an integer.

According to Lemma $2^{\prime}, \sum_{k=0}^{t} \omega_{k}^{(\alpha)}>0$, for $t \geq 0$ is an integer and $0<\alpha<1$.

Here, $t$ can be considered as the support length of the truncated G-L formula. When $t_{1}>t_{2}$, where $t_{1}$ and $t_{2}$ are two support lengths and $t_{1} \geq 0$ and $t_{2} \geq 0$, we have

$$
\sum_{k=0}^{t_{1}} \omega_{k}^{(\alpha)}<\sum_{k=0}^{t_{2}} \omega_{k}^{(\alpha)} .
$$

Lemma 12. The absolute values of truncated errors for $s(x)=1$ become smaller as the fractional orders $0<\alpha<1$ become larger.

Proof. Since $\omega_{0}^{(\alpha)}=1$ and $\omega_{1}^{(\alpha)}=-\alpha$, for $\alpha_{1}>\alpha_{2}, 0<\alpha_{1}<1$, and $0<\alpha_{2}<1$, where $\alpha_{1}$ and $\alpha_{2}$ are two fractional orders, we have

$$
1-\alpha_{1}>0, \quad 1-\alpha_{2}>0, \quad 1-\alpha_{1}<1-\alpha_{2} .
$$

According to Lemma 2 , then $\omega_{k}^{(\alpha)}=(1-(\alpha+1) / k) \omega_{k-1}^{(\alpha)}$. Thus,

For $0<\alpha<1$ and $k \geq 2$, we have $0<(1-(\alpha+1) / k)<1$.

$$
\omega_{k}^{(\alpha)}<0, \quad\left|\omega_{k}^{(\alpha)}\right|<\left|\omega_{k-1}^{(\alpha)}\right|, \quad \omega_{k}^{(\alpha)}>\omega_{k-1}^{(\alpha)},
$$

where $|\cdot|$ represents the absolute value. Thus, for $\alpha_{1}>\alpha_{2}$, $0<\alpha_{1}<1$, and $0<\alpha_{2}<1$, we have

$$
0<\left(1-\frac{\alpha_{1}+1}{k}\right)<\left(1-\frac{\alpha_{2}+1}{k}\right)<1
$$

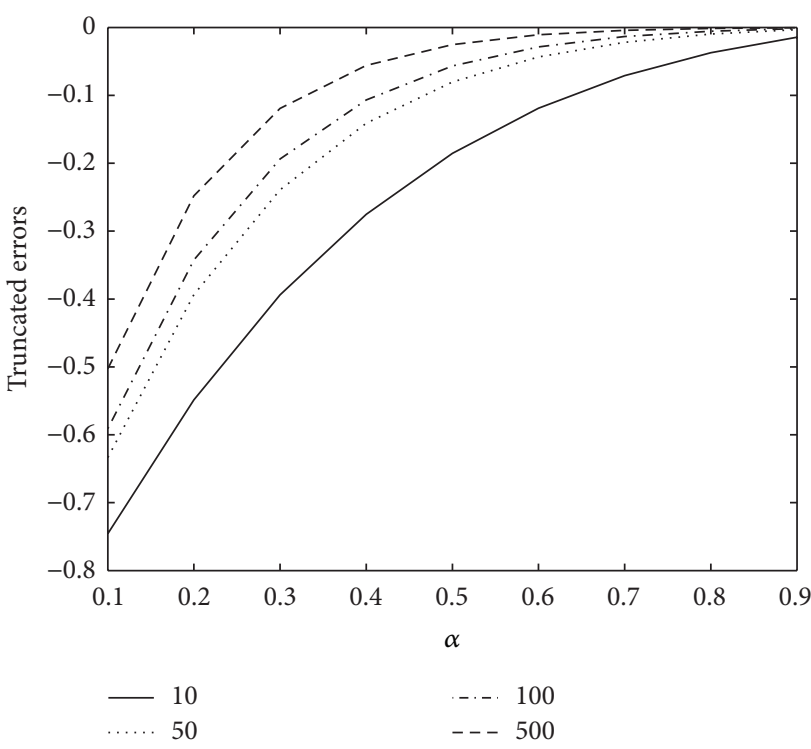

FIGURE 1: Truncated errors of truncated G-L formula with support lengths $10,50,100$, and 500 and fractional orders $\alpha$ from 0.1 to 0.9 .

If $0>\omega_{k}^{\left(\alpha_{1}\right)}>\omega_{k}^{\left(\alpha_{2}\right)}$, for $k \geq 1, \alpha_{1}>\alpha_{2}, 0<\alpha_{1}<1$, and $0<\alpha_{2}<1$.

Thus, we can assume that $\alpha_{1}=\alpha_{2}+c_{1}, 1>c_{1}>0$, and $\omega_{k}^{\left(\alpha_{1}\right)}=\omega_{k}^{\left(\alpha_{2}\right)}+c_{2}, c_{2}>0$. We have

$$
\begin{aligned}
\omega_{k+1}^{\left(\alpha_{1}\right)}= & \omega_{k}^{\left(\alpha_{1}\right)}\left(1-\frac{\alpha_{1}+1}{k}\right) \\
= & \left(\omega_{k}^{\left(\alpha_{2}\right)}+c_{2}\right)\left(1-\frac{\alpha_{2}+1+c_{1}}{k}\right) \\
= & \omega_{k}^{\left(\alpha_{2}\right)}\left(1-\frac{\alpha_{2}+1}{k}\right)+\omega_{k}^{\left(\alpha_{2}\right)}\left(-\frac{c_{1}}{k}\right) \\
& +c_{2}\left(1-\frac{\alpha_{2}+1+c_{1}}{k}\right) \\
= & \omega_{k+1}^{\left(\alpha_{2}\right)}+\omega_{k}^{\left(\alpha_{2}\right)}\left(-\frac{c_{1}}{k}\right)+c_{2}\left(1-\frac{\alpha_{2}+1+c_{1}}{k}\right)
\end{aligned}
$$

Since $\omega_{k}^{\left(\alpha_{2}\right)}\left(-c_{1} / k\right)>0$ and $c_{2}\left(1-\left(\alpha_{2}+1+c_{1}\right) / k\right)>0$, we have

$$
\omega_{k+1}^{\left(\alpha_{1}\right)}>\omega_{k+1}^{\left(\alpha_{2}\right)}
$$

Thus, $\sum_{k=1}^{t} \omega_{k}^{\left(\alpha_{2}\right)}<\sum_{k=1}^{t} \omega_{k}^{\left(\alpha_{1}\right)}<0$ and $\sum_{k=0}^{t} \omega_{k}^{\left(\alpha_{2}\right)}>$ $\sum_{k=0}^{t} \omega_{k}^{\left(\alpha_{1}\right)}>0$.

Summary of above two lemmas: we have that the long support and large fractional orders of truncated G-L formulas will have small absolute values of truncated errors. Experiments shown in Table 1 and Figure 1 also support these theory analysis results. Note that the truncated errors are negative numbers. Therefore, discussing their absolute values can show the differences between zeros and errors.

5.2. Experiments. In order to test if the new truncated method can reduce the truncated errors in real signals, two 
TABLE 1: Truncated errors of truncated left-handed G-L formula for $s(x)=1$ with support lengths from 10 to 500 (rows) and fractional-orders $\alpha$ from 0.1 to 0.9 (columns).

\begin{tabular}{llllllllll}
\hline $\begin{array}{l}\text { Length of } \\
\text { support }\end{array}$ & $\alpha=0.1$ & $\alpha=0.2$ & $\alpha=0.3$ & $\alpha=0.4$ & $\alpha=0.5$ & $\alpha=0.6$ & $\alpha=0.7$ & $\alpha=0.8$ & $\alpha=0.9$ \\
\hline 10 & -0.7475 & -0.5487 & -0.3939 & -0.2752 & -0.1855 & -0.1190 & -0.0710 & -0.0372 & -0.0145 \\
30 & -0.6672 & -0.4368 & -0.2795 & -0.1739 & -0.1043 & -0.0595 & -0.0315 & -0.0147 & -0.0051 \\
50 & -0.6335 & -0.3937 & -0.2392 & -0.1412 & -0.0804 & -0.0435 & -0.0219 & -0.0097 & -0.0032 \\
100 & -0.5908 & -0.3432 & -0.1939 & -0.1067 & -0.0566 & -0.0286 & -0.0134 & -0.0055 & -0.0017 \\
200 & -0.5510 & -0.2979 & -0.1573 & -0.0808 & -0.0400 & -0.0188 & -0.0082 & -0.0032 & -0.0009 \\
500 & -0.5027 & -0.2479 & -0.1194 & -0.0559 & -0.0253 & -0.0108 & -0.0043 & -0.0015 & -0.0004 \\
\hline
\end{tabular}

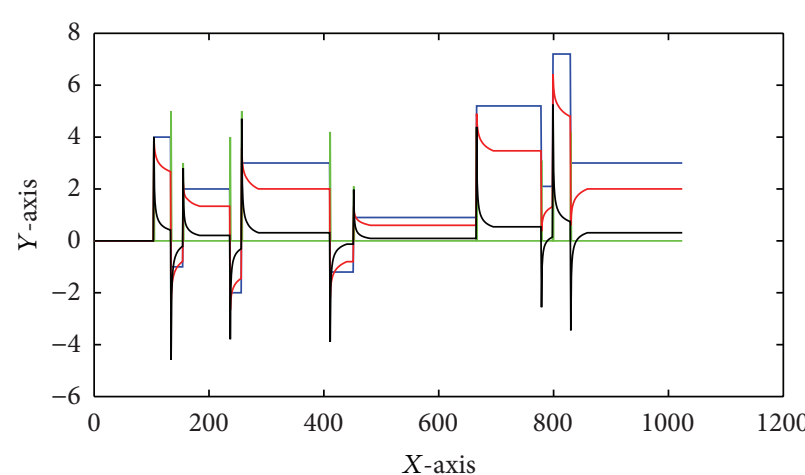

(a)

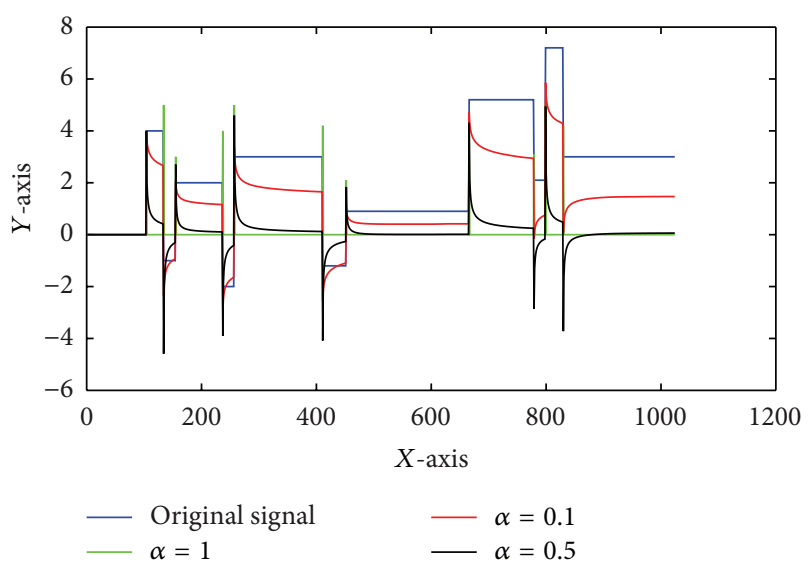

(c)

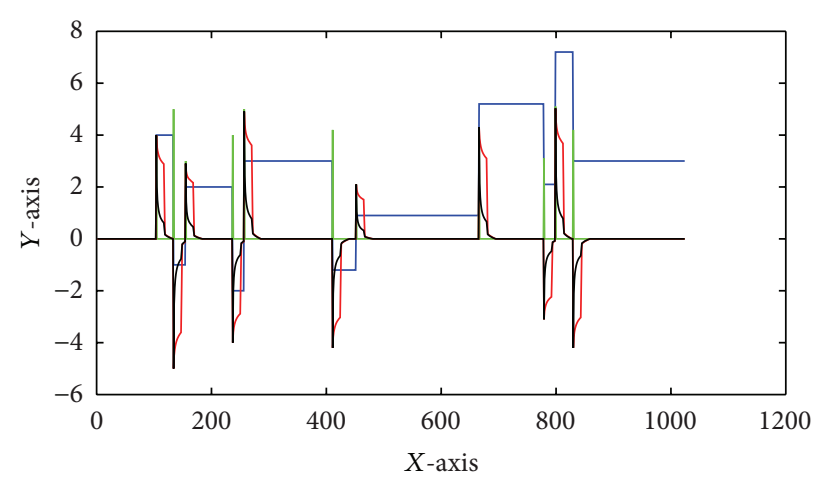

(b)

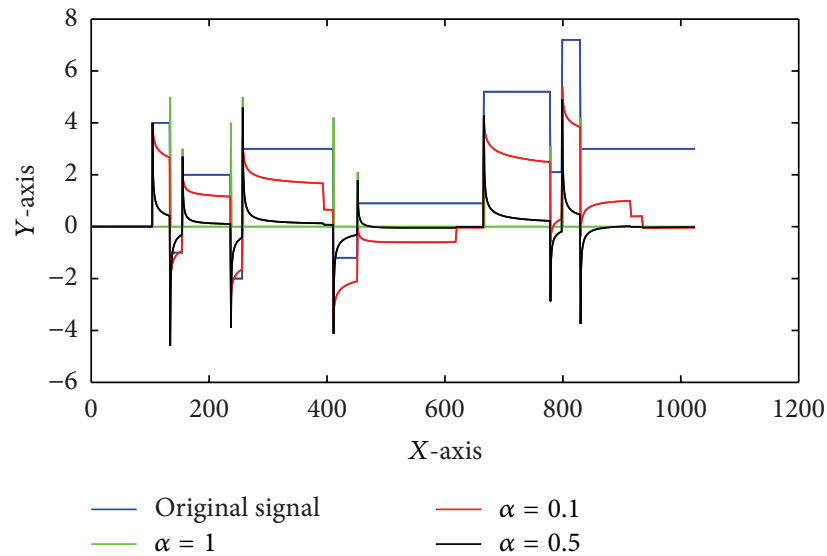

(d)

FIGURE 2: The original signal (blocks with 1024 samples, blue lines), its fractional derivatives of truncated G-L with different fractional orders ((a) and (c)), and its fractional derivatives of the new method with different fractional orders ((b) and (d)), where the support length of (a) and (b) is 30 and the support length of (c) and (d) is 500.

test signals, blocks, and bumps, with 1024 samples, are used for analysis of the performance of our new framework (see Figures 2 and 3 ).

From Figure 2, we can see both integer-order derivatives (green lines) and fractional derivatives $(\alpha=0.1$ are represented by red lines and $\alpha=0.5$ are represented by black lines) have the properties that the singularities are related to the local extrema. Just as discussed above, the values of truncated G-L formula are not zeros in smooth regions (see Figures 2(a) and 2(c)). Moreover, coinciding Lemma 11, the signals with longer truncated length will be nearer to zeros in the smooth segments than the shorter length signals. The bigger fractional orders will also have better performance in sharper impulses in singularities and much more near zeros in the smooth segments than the smaller fractional orders, which coincides Lemma 12 (see Figures 2(a) and 2(c)).

When truncated length $t=30$ for the new definition of fractional derivatives (see Figure 2(b)), the values of new definition at singularities have very high impulses comparing to the corresponding truncated G-L formula (Figure 2(a)), which is a very impressive nature to detect, locate, and preserve singularities. Moreover, near singularities, the values 


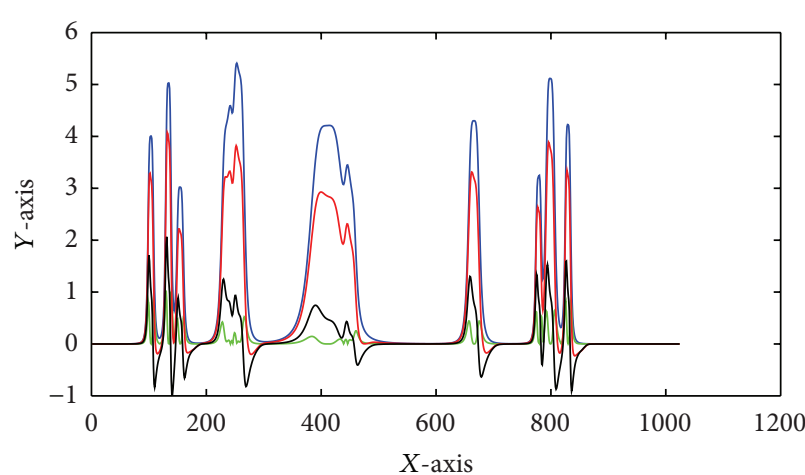

(a)

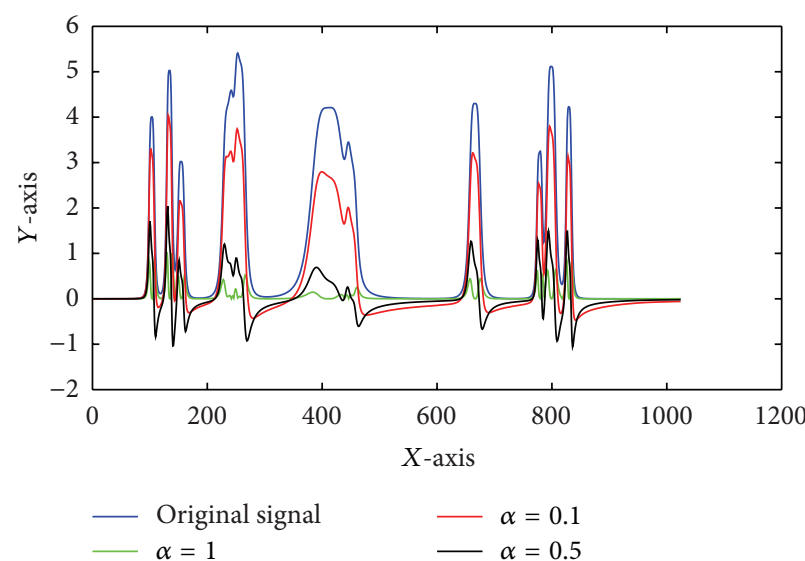

(c)

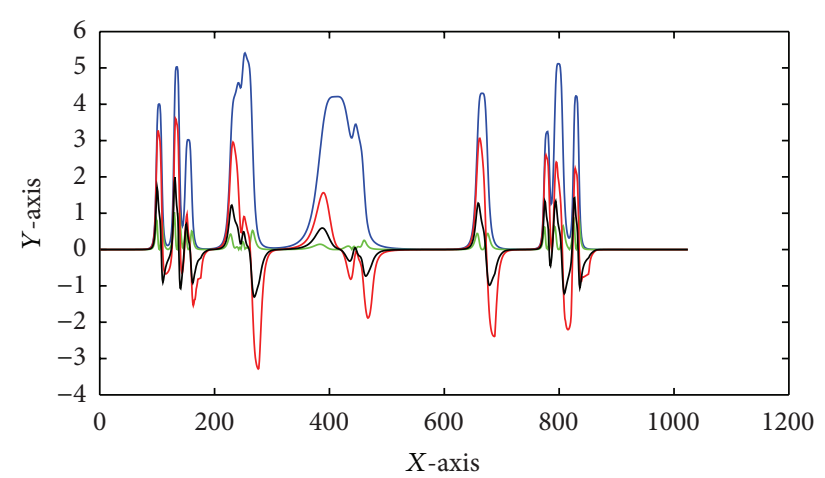

(b)

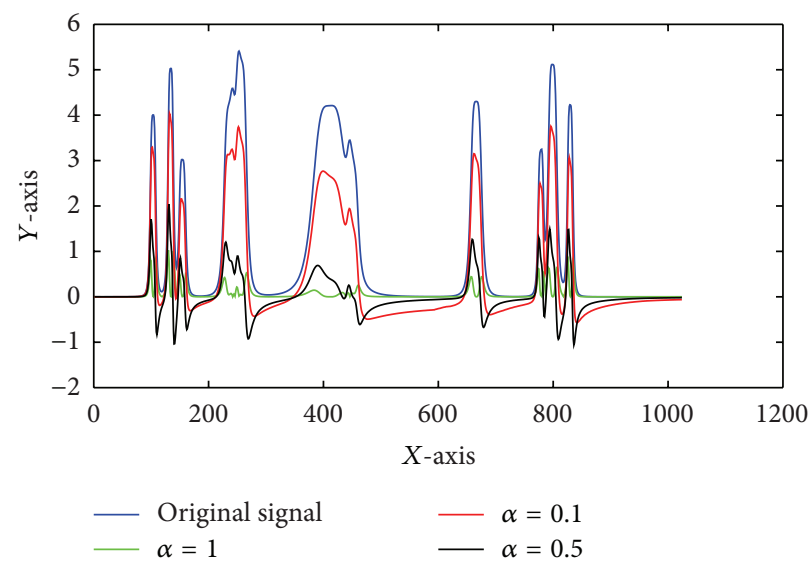

(d)

FIGURE 3: The original signal (bumps with 1024 samples, blue line), its fractional derivatives of truncated G-L with different fractional orders ((a) and (c)), and its fractional derivatives of the new method with different fractional orders ((b) and (d)), where the support length of (a) and (b) is 30 and the support length of (c) and (d) is 500.

are decreased/increased gradually to zeros and the values of the smooth segments are zeros. These characters together ensure that the new fractional derivatives can obtain good results in signal processing.

Observing Figure 3, we can find that 1-order derivatives cannot locate "bump like" singularities: (1) there are two extremums of 1-order derivatives even for a very narrow bump, for example, the first bump; (2) some weak singularities cannot be detected, for example, the left singularity of the fifth bump.

Fortunately, all fractional derivatives have better performance in above two sides. That is, each narrow bump only has one extremum of the fractional derivatives.

The best performance of the new definition for block signal is the derivative with truncated length $t=30$ and fractional order $\alpha=0.1$, which also has the best natures for the bump signal. That is, it has very high impulses in singularities and equals to zeros in smooth segments. Weak singularity in the left of the fifth bump has a very obvious high impulse, which can be detected and located easily. It is very interesting thing that the weak impulses in two signals using truncated G-L formula are enhanced by new definitions of fractional derivatives with small $\alpha$ and $t$ by comparing Figures 2(a) and 2(b) and Figures 3(a) and 3(b).

In summary, from the simulation results of two test signals, we can conclude that the new definition of fractional derivatives has the best performance in three type derivatives including 1-order derivatives, truncated G-L formula and themselves.

\section{Conclusions}

In this paper, we study errors of truncated GrünwaldLetnikov formula with $0<\alpha<1$ and then the errors are corrected by the medians of remainder parts of signals, which has some very impressive natures in signal processing. That is, it has very high impulses in singularities, which can detect and locate singularities easily; the values of the new definition are equal to zeros in smooth segments, which can be used efficiently in signal smoothing and filtering. Moreover, it also has good performance in weak singularity detection and location. 


\section{Conflict of Interests}

The author declares that there is no conflict of interests regarding the publication of this paper.

\section{Acknowledgments}

This paper is supported by the National Natural Science Foundation of China (nos. 60873102 and 60873264), Major State Basic Research Development Program (no. 2010CB732501), and Open Foundation of Visual Computing and Virtual Reality Key Laboratory of Sichuan Province (no. J2010N03). This work was supported by a grant from the National High Technology Research and Development Program of China (no. 2009AA12Z140).

\section{References}

[1] S. Hu, Z. Liao, D. Sun, and W. Chen, "A numerical method for preserving curve edges in nonlinear anisotropic smoothing," Mathematical Problems in Engineering, vol. 2011, Article ID 186507, 14 pages, 2011.

[2] Y. Chen, Y. Li, W. Yu, L. Luo, W. Chen, and C. Toumoulin, "Jointmap tomographic reconstruction with patch similarity based mixture prior model," Multiscale Modeling \& Simulation, vol. 9, no. 4, pp. 1399-1419, 2011.

[3] Y. Chen, X. Yin, L. Shi et al., "Improving abdomen tumor low-dose CT images using a fast dictionary learning based processing," Physics in Medicine and Biology, vol. 58, pp. 58035820, 2013.

[4] Z. Bian, J. Ma, J. Huang et al., "SR-NLM: a sinogram restoration induced non-local means image filtering for low-dose computed tomography," Computerized Medical Imaging and Graphics, vol. 37, no. 4, pp. 293-303, 2013.

[5] J. Ma, H. Zhang, Y. Gao et al., "Iterative image reconstruction for cerebral perfusion CT using a pre-contrast scan induced edgepreserving prior," Physics and Medical Biology, vol. 57, pp. 75197542, 2012.

[6] Z. Liao, "Low-dosed x-ray computed tomography imaging by regularized fully spatial fractional-order Perona-Malik diffusion," Advances in Mathematical Physics, vol. 2013, Article ID 371868, 9 pages, 2013.

[7] S. Hu, Z. Liao, and W. Chen, "Sinogram restoration for lowdosed X-ray computed tomography using fractional-order Perona-Malik diffusion," Mathematical Problems in Engineering, vol. 2012, Article ID 391050, 13 pages, 2012.

[8] Z. Jun and W. Zhihui, "A class of fractional-order multi-scale variational models and alternating projection algorithm for image denoising," Applied Mathematical Modelling. Simulation and Computation for Engineering and Environmental Systems, vol. 35, no. 5, pp. 2516-2528, 2011.

[9] J. Bai and X.-C. Feng, "Fractional-order anisotropic diffusion for image denoising," IEEE Transactions on Image Processing, vol. 16, no. 10, pp. 2492-2502, 2007.

[10] M. Janev, S. Pilipović, T. Atanacković, R. Obradović, and N. Ralević, "Fully fractional anisotropic diffusion for image denoising," Mathematical and Computer Modelling, vol. 54, no. 1-2, pp. 729-741, 2011.

[11] C. Wang, L. Lan, and S. Zhou, "Grunwald-Letnikov based adaptive fractional differential algorithm on image texture enhancing," Journal of Computational Information Systems, vol. 9, no. 2, pp. 445-454, 2013.

[12] H. A. Jalab and R. W. Ibrahim, "Texture enhancement based on the Savitzky-Golay fractional differential operator," Mathematical Problems in Engineering, vol. 2013, Article ID 149289, 8 pages, 2013.

[13] S. Hu, "External fractional-order gradient vector Perona-Malik diffusion for sinogram restoration of low-dosed x-ray computed tomography," Advances in Mathematical Physics, vol. 2013, Article ID 516919, 10 pages, 2013.

[14] R. Hilfer, Applications of Fractional Calculus in Physics, World Scientific Publishing, River Edge, NJ, USA, 2000.

[15] S. Hu and P. Liang, "Theory analysis of left-handed GrnwaldLetnikov formula with $0<\alpha<1$ to detect and locatesingularities," Abstract and Applied Analysis. In press.

[16] W.-S. Chen, C. Zhang, and S. Y. Chen, "Geometric distribution weight information modeled using radial basis function with fractional order for linear discriminant analysis method," Advances in Mathematical Physics, vol. 2013, Article ID 825861, 9 pages, 2013.

[17] W.-S. Chen, P. C. Yuen, and X. Xie, "Kernel machine-based rank-lifting regularized discriminant analysis method for face recognition," Neurocomputing, vol. 74, no. 17, pp. 2953-2960, 2011.

[18] M. Li, "A class of negatively fractal dimensional Gaussian random functions," Mathematical Problems in Engineering, vol. 2011, Article ID 291028, 18 pages, 2011.

[19] M. Li and W. Zhao, "On bandlimitedness and lag-limitedness of fractional Gaussian noise," Physica A: Statistical Mechanics and its Applications, vol. 392, no. 9, pp. 1955-1961, 2013.

[20] M. Li, S. C. Lim, C. Cattani, and M. Scalia, "Characteristic roots of a class of fractional oscillators," Advances in High Energy Physics, vol. 2013, Article ID 853925, 7 pages, 2013.

[21] I. Podlubny, Fractional Differential Equations, vol. 198 of Mathematics in Science and Engineering, Academic Press, San Diego, Calif, USA, 1999.

[22] S. G. Samko, A. A. Kilbas, and O. I. Marichev, Fractional Integrals and Derivatives: Theory and Applications, Gordon and Breach Science Publishers, London, UK, 1993.

[23] E. Sousa, "How to approximate the fractional derivative of order $1<\alpha \leq 2$," International Journal of Bifurcation and Chaos in Applied Sciences and Engineering, vol. 22, no. 4, 13 pages, 2012. 


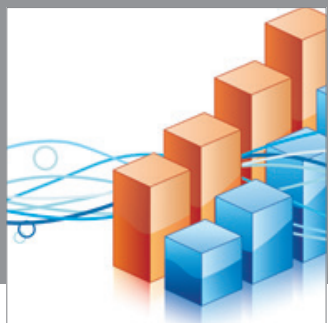

Advances in

Operations Research

mansans

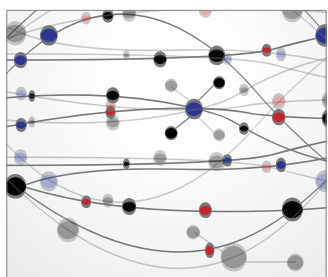

The Scientific World Journal
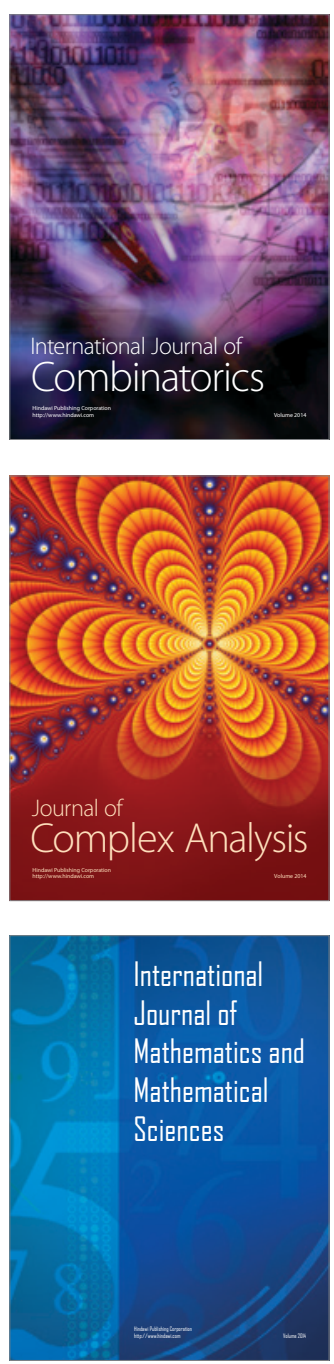
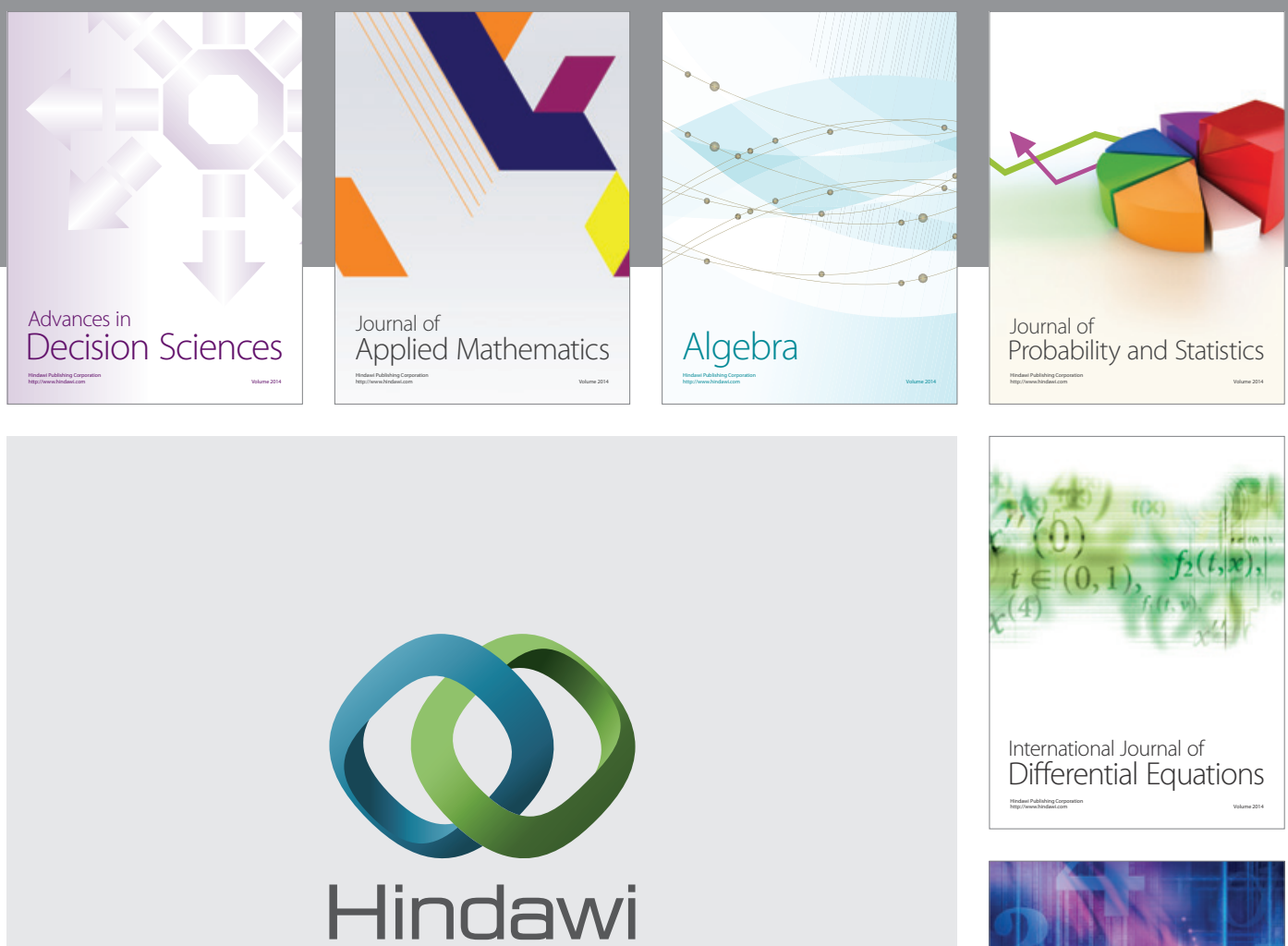

Submit your manuscripts at http://www.hindawi.com
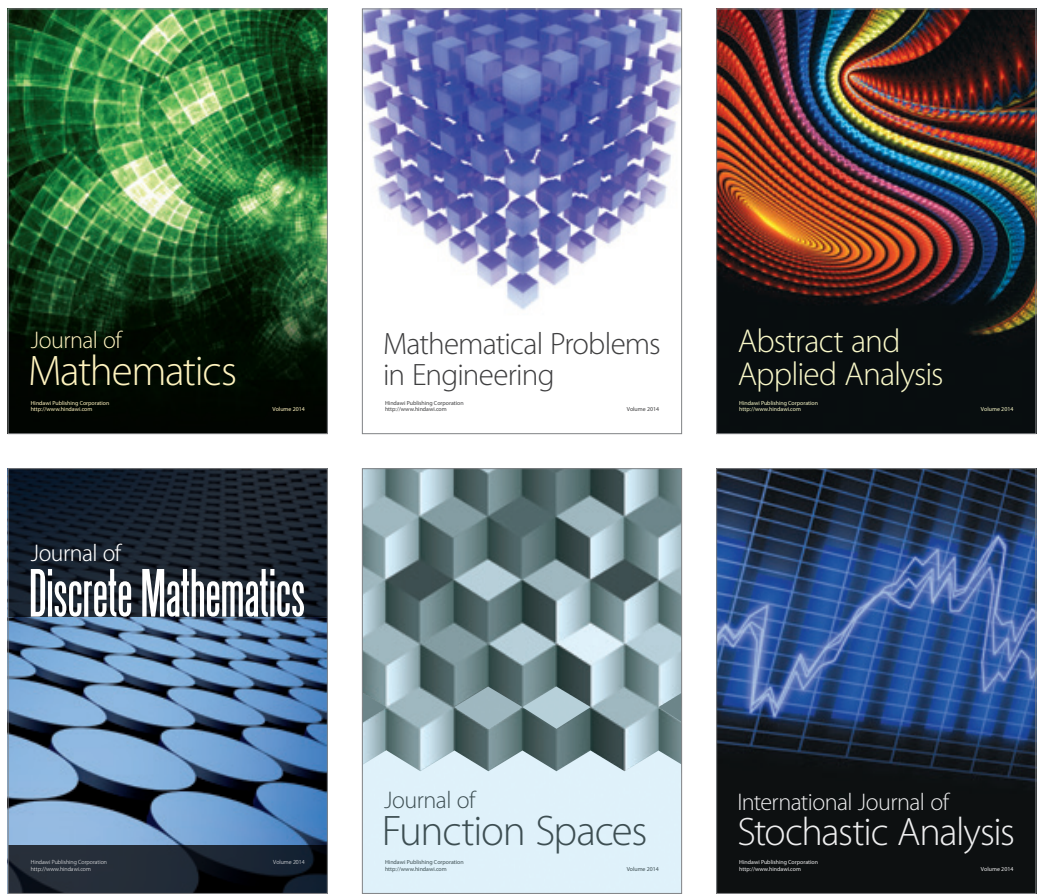

Journal of

Function Spaces

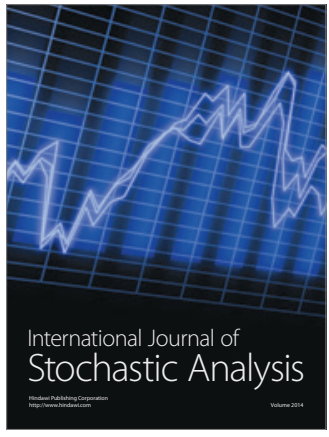

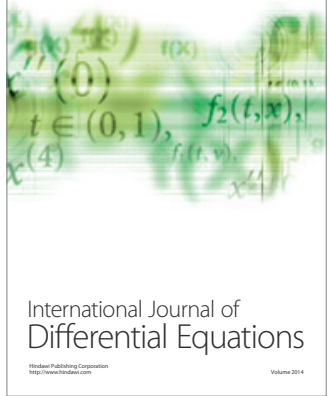
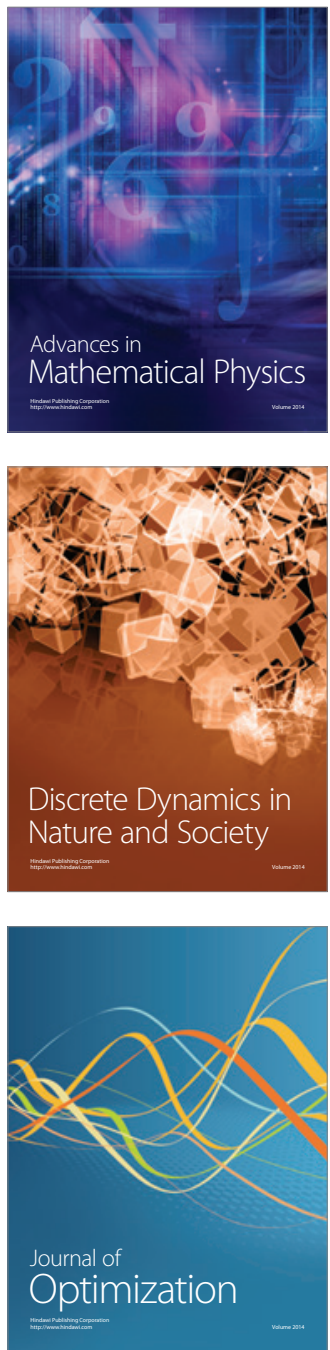